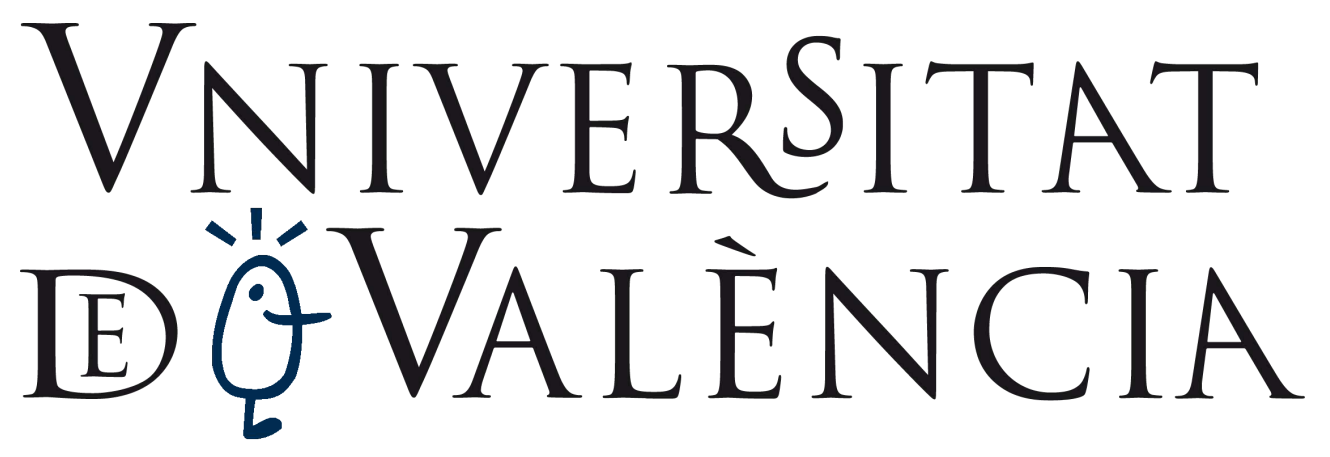

Copyright $\odot 2010$ The Institute of Electronics, Information and Communication Engineers

Citation for the original published paper:

Jingya LI, Xiaodong XU, Xin CHEN, Xiaofeng TAO, Hui ZHANG, Tommy SVENSSON, Carmen BOTELLA, Downlink Radio Resource Allocation for Coordinated Cellular OFDMA Networks, IEICE Transactions on Communications, 2010, Volume E93.B, Issue 12, Pages 3480-3488, Released December 01, 2010,

Online ISSN 1745-1345, Print ISSN 0916-8516

https://doi.org/10.1587/transcom.E93.B.3480

Publisher version can be found in:

https://www.jstage.jst.go.jp/article/transcom/E93.B/12/E93.B_12_3480/_article/-char/en

IEICE Transactions on Communications home page:

https://www.ieice.org/cs/jpn/EB/index.html 


\title{
Downlink Radio Resource Allocation for Coordinated Cellular OFDMA Networks
}

\author{
Jingya $\mathrm{LI}^{\dagger a)}$, Xiaodong $\mathrm{XU}^{\dagger}$, Xin $\mathrm{CHEN}^{\dagger}$, Xiaofeng $\mathrm{TAO}^{\dagger}$, Nonmembers, Hui $\mathrm{ZHANG}^{\dagger}$, Member, \\ Tommy SVENSSON ${ }^{\dagger \dagger}$, and Carmen BOTELLA ${ }^{\dagger \dagger}$, Nonmembers
}

\begin{abstract}
SUMMARY Base station coordination is considered as a promising technique to mitigate inter-cell interference and improve the cell-edge performance in cellular orthogonal frequency division multiple-access (OFDMA) networks. The problem to design an efficient radio resource allocation scheme for coordinated cellular OFDMA networks incorporating base station coordination has been only partially investigated. In this contribution, a novel radio resource allocation algorithm with universal frequency reuse is proposed to support base station coordinated transmission. Firstly, with the assumption of global coordination between all base station sectors in the network, a coordinated subchannel assignment algorithm is proposed. Then, by dividing the entire network into a number of disjoint coordinated clusters of base station sectors, a reduced-feedback algorithm for subchannel assignment is proposed for practical use. The utility function based on the user average throughput is used to balance the efficiency and fairness of wireless resource allocation. System level simulation results demonstrate that the reduced-feedback subchannel assignment algorithm significantly improves the cell-edge average throughput and the faimess index of users in the network, with acceptable degradation of cell-average performance.

key words: base station coordination, OFDMA, radio resource allocation,
\end{abstract} utility function

\section{Introduction}

Driven by the need to support data applications at higher throughputs and spectral efficiency, orthogonal frequency division multiplexing (OFDM) based multiple access is being considered as a promising multiple access method for next generation wireless networks. OFDMA has been adopted as the downlink access technology of 3rd generation partnership project (3GPP) long term evolution (LTE) and LTE-Advanced standards [1], [2]. Based on the OFDM technique, OFDMA inherits the immunity to intra-cell interference. However, inter-cell interference (ICI) is still a major issue. In fact, a frequency reuse factor equal to one causes serious ICI to users at the cell-edge areas, leading to poor cell-edge throughputs. Therefore, ICI is a factor causing significant performance and fairness degradation in the network [3].

\footnotetext{
Manuscript received May 1, 2010.

Manuscript revised August 1, 2010.

${ }^{\dagger}$ The authors are with Wireless Technology Innovation Institute, Key Laboratory of Universal Wireless Communication, Ministry of Education, Beijing University of Posts and Telecommunications (BUPT), Beijing, China.

${ }^{\dagger}$ The authors are with the Department of Signals and Systems, Chalmcrs Univcrsity of Technology, SE-412-96 Gothenburg, Sweden.

a) E-mail: liji@chalmers.se

DOI: 10.1587 /transcom.E93.B.3480
}

In conventional aggressive reuse cellular systems, ICI can be mitigated via advanced receiver processing, or rejection in the spatial and other domains [4], [5]. In the downlink, however, receiver processing necessarily burdens the mobile station (MS) by adding complexity, and mobile equipment may have only limited signal processing capabilities due to the cost and battery life constraints.

Recently, base station coordination (BSC), also known as network coordination, has emerged as a means to mitigate ICI and further improve the cell-edge performance [6][9]. In a coordinated cellular network, base stations (BSs) are inter-connected via a high-speed backbone and all base antennas act together as a single network antenna array. A central unit (CU) is assumed to determine the channel assignment for users and the power allocation for subcarriers throughout the entire network. Coordinated BSs not only share channel state information (CSI) but also the data to be transmitted to the MS. The ICI is then reduced by using the signals transmitted from other cells to assist the transmission instead of acting as interference.

Clearly, radio resource allocation plays an important role in optimizing the performance of coordinated cellular OFDMA networks. Early studies have mainly focused on the single-cell case, where the resource allocation problem in multi-user OFDMA systems is a combinatorial problem whose complexity increases exponentially with the number of subchannels and the number of users. By introducing time-sharing or frequency-sharing variables, the problem is solved through convex relaxation methods, see for example [10]-[12]. More recently, research has shifted towards the multi-cell coordinated resource allocation case with one BS transmission, where the resource allocation is coordinated between several cells to decrease the ICI. A semi-distributed resource allocation scheme is proposed in [13], where the specific set of traffic channels of each cell is decided at a super-frame level and the per-cell optimization is performed at the frame level. However, the authors consider only one strong interferer and do not consider the fairness issues, such as cell-edge performance. In [14], M.C. Necker proposes a distributed interference coordination scheme by separating the global optimization problem into two separate problems and solving these problems based on graph coloring algorithms. A heuristic algorithm to distribute radio resources among multiple users according to their individual QoS requirements is introduced in [15]. In [16] and [17], utility functions based multi-cell coordinated resource allocation 
schemes are proposed. Note that one key assumption of [13]-[17] is that each MS can only communicate with a single BS, which is called the anchor (or serving) BS. Hence, these proposed resource allocation schemes cannot support base station coordinated transmission, since base station coordinated transmission needs a group of BSs to concurrently transmit signals to one MS.

In [18], a two-phase channel-assignment method is proposed considering BSC, where ICI coordination and BSC are solved via graph theory in the first phase and the subchannel allocation is accomplished in the second phase. To the best of our knowledge, this is the only reference paper that considers a multi-BS coordinated transmission case in its resource allocation scheme. However, the objective function designed for subchannel assignment in the second phase in [18] is not optimized for BSC, since the signal-tonoise ratio (SNR) of the MS is only derived from its serving BS.

In this paper, we propose a novel radio resource allocation algorithm to enable base station coordinated transmission in coordinated cellular OFDMA networks. Our focus is on OFDMA downlink with no intra-cell interference. We assume global coordination between all base station sectors in the network. A utility function based on user average throughput is introduced to formulate the optimization problem, since it can naturally balance the fairness and efficiency. The proposed scheme maximizes the sum utility function of users in the network and performs subchannel assignment for the coordinated cellular network.

Global coordination within the network can eliminate the ICI completely. However, in realistic cellular networks, only a limited number of BSs can cooperate in order to make the measurement and signaling overhead affordable [19][23]. Hence, by dividing the entire network into a number of static disjoint coordinated clusters, a reduced-feedback algorithm for subchannel assignment is proposed for practical implementation. System level simulation results demonstrate that the proposed algorithm can offer a substantial improvement in cell-edge performance and system fairness in aggressive frequency reuse cellular networks, with an acceptable degradation of cell-average performance.

The rest of this paper is organized as follows: In Sect. 2, we present the system model and formulate the maximum sum utility function problem. In Sect. 3 , by solving the optimization problem, the resource allocation algorithm is proposed. Firstly, we provide a coordinated subchannel assignment algorithm assuming global coordination within the network. Then, a reduced-feedback algorithm is proposed for practical use. Section 4 presents the system level simulation results. Conclusions and future work are presented in Sect. 5.

\section{System Model and Problem Formulation}

\subsection{System Model and SINR Derivation}

We consider the downlink of a coordinated cellular OFDMA

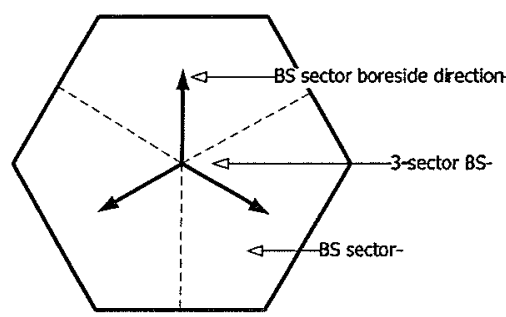

Fig. 1 An example of a 3-sector BS.

network with 3-sector BSs. Each BS sector (BSS) has one directional antenna and is associated with a directional sector area. The 3 antennas of each BS are located at the same site, which is illustrated in Fig. 1. Each MS is equipped with one receive antenna and assigned to a serving BSS that is selected based on long-term channel quality measurements. In coordinated cellular networks, each MS can be served by a set of BSSs with the same time and frequency resources. Similar to the diversity set defined in [18], a cooperative transmission set (CTS) is designed for each MS on each subchannel, which is formed by the serving BSS and neighboring BSSs that provide data transmission service to this MS on the corresponding subchannel. We assume global coordination within the network. Hence, the formation of the CTS for each MS is unconstrained, which means the BSSs contained in a CTS are selected from all the BSSs in the network.

Assume there are a total of $N$ BSSs within the network. Let $\mathcal{M}_{n}$ denote the MS set for BSS $n$. Let $M_{n}=\left|\mathcal{M}_{n}\right|$ denote the number of MSs in BSS $n$, where $\left|\mathcal{M}_{n}\right|$ is the cardinality of the set $\mathcal{M}_{n}$. Hence, the entire network has a total of a set of $M=\sum_{n=1}^{N} M_{n}$ users. $K$ subchannels are available for resource allocation and the frequency reuse factor is one, i.e., each BSS will use all $K$ subchannels. Note that each subchannel consists of twelve contiguous subcarriers. We assume a full traffic model wherein each BSS always has data available for transmission to all connected MSs. For simplicity, we do not consider space division multiple access (SDMA) techniques in this paper. Hence, each subchannel of a BSS can only be assigned to one MS.

In conventional cellular networks where each MS communicates with the serving BSS, the discrete-time baseband signal received by MS $m$ of BSS $i$ on subchannel $k$ is given by

$$
r_{m}^{k}=\underbrace{\sqrt{P_{i}^{k}} H_{i, m}^{k} s_{i}^{k}}_{\text {signal of interest }}+\underbrace{\sum_{j \neq i} \sqrt{P_{j}^{k}} H_{j, m}^{k} s_{j}^{k}}_{\text {inter-cell interference }}+\underbrace{n_{m}^{k}}_{\text {noise }}
$$

where $P_{i}^{k}$ is the transmitted power from BSS $i$ on subchannel $k$. For simplicity, $P_{i}^{k}$ is assumed to be constant, i.e., no power control is performed. $H_{i, m}^{k}$ is the complex channel response between BSS $i$ and MS $m$ on subchannel $k$, consisting of path loss, large-scale fading, and small-scale fading. $s_{i}^{k}$ is the complex symbol transmitted by BSS $i$ on subchannel $k$, which has zero mean and normalized power, i.e., 
$E\left(\left|s_{i}^{k}\right|^{2}\right)=1 . n_{m}^{k}$ is the additive white Gaussian noise with noise power $N_{0}$.

Let $G_{i, m}^{k}=\left|H_{i, m}^{k}\right|^{2}$, then the instantaneous signal-tointerference-plus-noise ratio (SINR) on subchannel $k$ for MS $m$ is determined as

$$
\gamma_{m}^{k}=\frac{P_{i}^{k} G_{i, m}^{k}}{\sum_{j \neq i} P_{j}^{k} G_{j, m}^{k}+N_{0}} .
$$

To obtain the SINR expression for coordinated cellular networks, note that, each MS can receive signals from more than one BSS. The CTS of MS $m$ on subchannel $k$ is denoted as $\mathcal{D}_{m}^{k}=\mathcal{A}_{m}+\mathcal{B}_{m}^{k}$, where $\mathcal{A}_{m}$ is the serving BSS set that has only one element, and $\mathcal{B}_{m}^{k}$ is the neighboring BSS set that may include zero, one, or multiple BSSs. Note that in global coordinated networks, the CTS is obtained over all the network, we have $\left|\mathcal{D}_{m}^{k}\right|<N$. Define $\overline{\mathcal{D}}_{m}^{k}$ to be the complement set of $\mathcal{D}_{m}^{k}$. Then, the instantaneous received SINR on subchannel $k$ for MS $m$ is given by

$$
\gamma_{m}^{k}=\frac{\sum_{i \in \mathcal{D}_{m}^{k}} P_{i}^{k} G_{i, m}^{k}}{\sum_{j \in \overline{\mathcal{D}}_{m}^{k}} P_{j}^{k} G_{j, m}^{k}+N_{0}}
$$

Finally, according to Shannon theorem, the achievable transmission rate of MS $m$ on subchannel $k$ can be expressed as

$$
R_{m}^{k}=B \log _{2}\left(1+\beta \gamma_{m}^{k}\right),
$$

where $B$ is the bandwidth of each subchannel, and $\beta$ is the SINR gap, which is a constant related to the target bit error rate (BER) given by [24]

$$
\beta=-\frac{1.5}{\ln (5 B E R)} \text {. }
$$

Hence, the instantaneous data transmission rate for MS $m$ at time $t$ becomes

$$
R_{m}(t)=\sum_{k=1}^{K} R_{m}^{k}(t)
$$

\subsection{Optimization Problem Formulation}

In this subsection, the coordinated resource allocation problem in the network with frequency reuse factor equal to one is described. The utility function of each MS is defined with respect to its average throughput. Let $U_{m}(\cdot)$ denote the utility function of MS $m$, which is non-decreasing and concave to balance the efficiency and faimess of the subchannel allocation. To make the optimization problem more tractable, we assume that the utility curve is continuously differentiable.

The optimization objective is to maximize the sum utility of the MSs in the entire network. Let $\mathcal{N}, \mathcal{M}$ and $\mathcal{K}$ denote the set of BSSs, MSs and subchannels, respectively.
Given a fixed power allocation, the optimization problem can be mathematically formulated as follows

$$
\begin{aligned}
& \max _{\mathcal{D}_{m}^{*}, m \in \mathcal{A}, k \in \mathcal{K}} \sum_{m=1}^{M} U_{m}\left(\bar{R}_{m}(t)\right) \\
& \text { subject to 1) } \mathcal{D}_{m}^{k} \subseteq \mathcal{N}, \forall k \in \mathcal{K} \text {, } \\
& \text { 2) } \mathcal{D}_{i}^{k} \cap \mathcal{D}_{j}^{k}=\phi, i \neq j, \forall i, j \in \mathcal{M}, \forall k \in \mathcal{K} \text {. }
\end{aligned}
$$

The average throughput of MS $m$ at time $t$ can be expressed using an exponentially low-pass time window as [25]

$$
\bar{R}_{m}(t)=\left(1-\rho_{w}\right) \bar{R}_{m}(t-1)+\rho_{u} R_{m}(t),
$$

where $\rho_{w}=\left(T_{s} / T_{w}\right), T_{s}$ is the slot length, and $T_{w}$ is the length of the window.

Note that the formation of the CTS for each user is included in the optimization problem. Constraint 1) means that the CTS of each MS is composed of a subset of BSSs of the network; constraint 2) means that the CTSs of all the MSs in the network are disjoint, which guarantees that a subchannel in a certain BSS is used by at most one MS.

\section{Coordinated Subchannel Assignment Algorithm}

In this section, the optimization problem of subchannel assignment in (7) is reformulated and solved based on the Lagrangian method by using a convex relaxation method, similar to the ones in Refs. [10]-[12]. Then a coordinated subchannel assignment algorithm is proposed for base station coordinated transmission, assuming global coordination with unconstrained CTS formation. Further, a reducedfeedback algorithm for subchannel assignment with constrained CTS formation is proposed for practical use.

\subsection{Optimization Problem Reformulation}

In a first step, we reformulate the above discrete allocation optimization problem as a nonlinear integer (0-1) programming one. Let $X=\left[x_{m, f(n, k)}\right]$ be the subchannel assignment matrix, where $f(n, k)$ denotes the subchannel $k$ of BSS $n$. $x_{m, f(n, k)}$ indicates whether subchannel $k$ of BSS $n$ is assigned to $\mathrm{MS} m$, that is

$$
x_{m, f(n, k)}= \begin{cases}1, & \text { if subchannel } k \text { of BSS } n \text { is assigned } \\ \text { to MS } m, \\ 0, & \text { otherwise. }\end{cases}
$$

Then, the CTS of MS $m$ on subchannel $k$ can be described as

$$
\mathcal{D}_{m}^{k}=\left\{n \mid x_{m, f(n, k)}=1, n \in \mathcal{N}\right\} .
$$

The SINR on subchannel $k$ for MS $m$ given by (3) can be described as

$$
\gamma_{m}^{k}=\frac{\sum_{i \in \mathcal{N}} P_{i}^{k} G_{i, m}^{k} x_{m, f(i, k)}}{\sum_{j \in \mathcal{N}}\left(P_{j}^{k} G_{j, m}^{k} \sum_{s \in \mathcal{M}, s \neq m} x_{s, f(j, k)}\right)+N_{0}} .
$$


Then, the equivalent nonlinear integer $(0-1)$ programming problem can be formulated as follows:

$$
\begin{array}{ll}
\max _{X} & U(X)=\sum_{m=1}^{M} U_{m}\left(\bar{R}_{m}(t)\right) \\
\text { subject to } & \text { 1) } \sum_{m \in \mathcal{M}} x_{m, f(n, k)}=1, \forall n \in \mathcal{N}, \forall k \in \mathcal{K}, \\
& \text { 2) } x_{m, f(n, k)} \in\{0,1\}, \forall m \in \mathcal{M}, \forall n \in \mathcal{N}, \\
& \forall k \in \mathcal{K} .
\end{array}
$$

It is straightforward to notice that the optimization problem in (12) is a combinatorial problem. To make the problem tractable, we relax the requirement $x_{m, f(n, k)} \in\{0,1\}$ to allow $x_{m, f(n, k)}$ being a real number within the interval $[0$, 1] to form a convex set. Notice that $x_{m, f(n, k)}$ can be considered as a time sharing factor of the subchannel $k$ in BSS $n$. Based on (8) and using the first-order Taylor expansion, the objective function in (12) can be rewritten as [25]

$$
U(X)=\sum_{m=1}^{M} U_{m}^{\prime}\left(\bar{R}_{m}(t-1)\right) R_{m}(t)
$$

which maximizes the sum of weighted throughputs. Note that $\bar{R}_{m}(t-1)$ is fixed at time $t$. From now on, $U_{m}^{\prime}$ is used to represent $U_{m}^{\prime}\left(\bar{R}_{m}(t-1)\right)$.

\subsection{Optimization Problem Solving}

Based on standard optimization theory, from (12) and (13), we obtain the Lagrangian

$$
L=\sum_{m=1}^{M}\left(U_{m}^{\prime} \sum_{k=1}^{K} R_{m}^{k}\right)-\sum_{n=1}^{N} \sum_{k=1}^{K} \lambda_{n, k}\left(\sum_{m=1}^{M} x_{m, f(n, k)}-1\right),
$$

where $\lambda_{n, k}$ is the Lagrangian multiplier for the constraint.

After differentiating $L$ with respect to $x_{m, f(n, k)}$, we obtain the necessary conditions for the optimal solution $x_{m, f(n, k)}^{*}$, specifically, if $x_{m, f(n, k)}^{*} \neq 0$, we have

$$
\begin{aligned}
& \left.\frac{\partial L}{\partial x_{m, f(n, k)}}\right|_{x_{m, f(n, k)=x_{m, f(n, h)}^{*}}}=\frac{B}{\ln 2}\left(U_{m}^{\prime} \omega_{m, n}^{k}-\sum_{i=\mathcal{M}, i \neq m} U_{i}^{\prime} \omega_{i, n}^{k} \gamma_{i}^{k}\right) \\
& -\lambda_{n, k} \begin{cases}=0 \text { if } x_{m, f(n, k)}^{*} \in(0,1), \\
>0 \text { if } x_{m, f(n, k)}^{*}=1,\end{cases}
\end{aligned}
$$

where

$$
\begin{aligned}
\omega_{i, n}^{k} & =\frac{\beta p_{n}^{k} G_{n, i}^{k}}{\left(1+\beta \gamma_{i}^{k}\right)\left(1+\sum_{j \in \mathcal{N}} p_{j}^{k} G_{j, i}^{k} \sum_{s \in \mathcal{M}, s \neq i} x_{s, f(j, k)}\right)} \\
& =\frac{\beta}{\left(1 / \gamma_{i}^{k}+\beta\right)} \times \frac{p_{n}^{k} G_{n, i}^{k}}{\sum_{j \in \mathcal{N}} p_{j}^{k} G_{j, i}^{k} x_{i, f(j, k)}} .
\end{aligned}
$$

Hence, we can conclude that

$$
x_{m, f(n, k)}^{*}= \begin{cases}(0,1) & \text { if } \lambda_{n, k}>\frac{B T_{m, n, k}}{\ln 2}, \\ 1 & \text { if } \lambda_{n, k}<\frac{B T_{m, n, k}}{\ln 2},\end{cases}
$$

where

$$
T_{m, n, k}=U_{m}^{\prime} \omega_{m, n}^{k}-\sum_{i=\mathcal{M}, i \neq m} U_{i}^{\prime} \omega_{i, n}^{k} \gamma_{i}^{k} .
$$

Since the integer constraint that a subchannel can only be used by one user must be satisfied, a reasonable integer solution based on (17) is provided. In particular, for a certain subchannel $k$ of BSS $n$, if $T_{m, n, k}$ for $m=1,2, \ldots, M$ are different, then only the user with the maximum $T_{m, n, k}$ can use that subchannel. In other words, for subchannel $k$ of BSS $n$, the subchannel assignment is

$$
\hat{m}(n, k)=\underset{m}{\arg \max } T_{m, n, k},
$$

where $\hat{m}(n, k)$ represents that the subchannel $k$ of BSS $n$ should be assigned to user $\hat{m}$.

Then, $T_{m, n, k}$ can be rewritten as

$$
T_{m, n, k}=\underbrace{U_{m}^{\prime} \omega_{m, n}^{k}}_{I^{1}}-\underbrace{\sum_{j \in \mathcal{N}, \hat{m}(j, k) \neq m} U_{\hat{m}(j, k)}^{\prime} \omega_{\hat{m}(j, k), j}^{k} \gamma_{\hat{m}(j, k)}^{k}}_{I^{2}},
$$

where $I^{1}$ represents the utility gained from assigning the subchannel to MS $m$, and $I^{2}$ represents the cost due to the interference introduced to other co-channel MSs.

According to Eq. (19), the subchannel $k$ of BSS $n$ should be assigned to the user with the maximum $T_{m, n, k}$. However, from Eq. (20), we can see that for a certain subchannel, the assignment decision made for one BSS is associated with the decision made by other BSSs, which is denoted as $I^{2}$. Hence, a suboptimal channel assignment strategy is proposed in this paper to maximize the sum of $T_{m, n, k}$ of all the BSSs on subchannel $k$ at time $t$, which is denoted as

$$
S_{k}^{t}=\arg \max \sum_{n=1}^{N}\left(U_{\hat{m}(n, k)}^{\prime} \omega_{\hat{m}(n, k), n}^{k}-\sum_{j \in \mathcal{N}, j \neq n} U_{\dot{m}(j, k)}^{\prime} \omega_{\hat{m}(j, k), j}^{k} \gamma_{\hat{m}(j, k)}^{k}\right)
$$

Therefore, the combinatorial optimization problem of $K$ subchannels is decomposed to $K$ independent optimization problems for each subchannel.

\subsection{Coordinated Subchannel Assignment Algorithm for BSC}

In this subsection, a coordinated subchannel assignment (CSA) algorithm is proposed assuming global coordination of all the BSSs in the network. The CSA algorithm is composed of two parts:

Initial Subchannel Assignment: In the above subsection, the combinatorial optimization problem of $K$ subchannels is decomposed to $K$ independent optimization problems for each subchannel as shown in Eq. (21). Hence, each subchannel is assigned according to the proposed suboptimal subchannel assignment strategy.

Iterative Subchannel Swapping: Iterative subchannel 
swapping is introduced to further increase the sum utility [26]. Let $\mathfrak{M}_{k}=\{\hat{m}(n, k)\}$ denote the set of MSs allocated with subchannel $k$ of all the BSSs in the network. $\Delta_{i, j}$ is defined as the incremental sum utility if assigning subchannel $i$ to MS set $\mathfrak{M}_{j}$ and assigning subchannel $j$ to MS set $\mathfrak{M}_{i}$. Then iterative swapping is done as follows:

- Step 1: For every pair of MS sets $\left(\mathfrak{M}_{i}, \mathfrak{M}_{j}\right)$ and the corresponding subchannels $(i, j)$, calculate the relevant $\triangle_{i, j}$

- Step 2: Among all pairs of MS sets, find $\left(i^{*}, j^{*}\right)=$ $\arg \max \Delta_{i, j}$.

- Step 3: if $\Delta_{i, j}>0$, swap subchannel $i$ from MS set $\mathfrak{M}_{i}$ to MS set $\mathfrak{M}_{j}$ and subchannel $j$ from $\mathfrak{M}_{j}$ to $\mathfrak{M}_{i}$.

- Step 4: Repeat Steps 1-3 until all $\Delta_{i, j}$ are negative.

\subsection{Reduced Feedback CSA-BSC Algorithm}

In the proposed CSA algorithm, global coordination between all BSSs in the network is assumed. In this case, the CTS for each MS on each subchannel is obtained over all the network. Therefore, each MS needs to estimate the full CSI from all BSSs and feed it back to the serving BSS in a per-slot time scale. In a second step, each BSS forwards this information to the CU. In frequency division duplex systems (FDD), this centralized framework requires an enormous amount of feedback and backhaul overhead. In addition, a high computational burden is caused in the CU. Therefore, a reduced-feedback CSA algorithm, named as CSA-BSC, is proposed in this section for practical implementations.

In order to decrease both the signaling and backhauling overhead, user grouping, e.g., serving only subsets of terminals with BSC, and clustering of base stations, i.e., dividing the network in small subsystems or cluster of BSSs, have been proposed [19]-[23]. In this paper, we use a static clustering technique, where the available BSSs are divided into a number of disjoint clusters of coordinated BSSs. Each cluster consists of three neighboring BSSs, and the formation of CTS is constraint within the cluster. Notice that BSC is independently applied in each cluster.

Assume that there is a CU for each cluster. Then, the resource allocation strategy in (21) can be applied locally in each cluster with some small modifications

$$
\begin{aligned}
S_{k}^{t}= & \arg \max \sum_{n \in \mathcal{N}_{G}}\left(U_{\hat{m}(n, k)}^{\prime} \omega_{\hat{m}(n, k), n}^{k}\right. \\
& \left.-\sum_{j \in \mathcal{N}_{G}, j \neq n} U_{\hat{m}(j, k)}^{\prime} \omega_{\hat{m}(j, k), j}^{k} \gamma_{\hat{m}(j, k)}^{k}\right),
\end{aligned}
$$

with

$$
\begin{aligned}
\omega_{i, n}^{k} & =\frac{\beta p_{n}^{k} G_{n, i}^{k}}{\left(1+\beta \gamma_{i}^{k}\right)\left(1+\sum_{j \in \mathcal{N}_{G}} p_{j}^{k} G_{j, i}^{k} \sum_{s \in \mathcal{M}_{G}, s \neq i} x_{s, f(j, k)}+\sum_{l \in \overline{\mathcal{N}}_{G}} p_{l}^{k} g_{l, i}\right)} \\
& =\frac{\beta}{\left(1 / \gamma_{i}^{k}+\beta\right)} \times \frac{p_{n}^{k} G_{n, i}^{k}}{\sum_{j \in N_{G}} p_{j}^{k} G_{j, i}^{k} x_{i, f(j, k)}}
\end{aligned}
$$

where $\mathcal{N}_{G}$ and $\mathcal{M}_{G}$ denote the set of BSSs and the set of MSs in the cluster, respectively. $g_{l, i}$ denotes the long-term gain from MS $i$ to BSS $j$, which only consists of path loss and large-scale fading.

Hence, the proposed CSA-BSC algorithm can be applied for each coordinated cluster in coordinated cellular networks.

\section{Performance Evaluation}

In this section, system level simulation is performed to evaluate the performance of the proposed resource allocation algorithm. Only the reduced-feedback algorithm is evaluated.

\subsection{Simulation Environment and Assumptions}

The structure of the clustered coordinated cellular network under study is shown in Fig. 2. It can be observed that each cluster is formed by 3 neighboring BSSs. A CU is allocated in each cluster.

In the simulations, users are uniformly dropped in each cell. As stated in the system model, equal power allocation is performed in all available subchannels. The natural logarithm function $\ln (\cdot)$ is used as the users' utility function, and the throughput filter window length, $T_{i j}$, is set to 100 slots. Table 1 lists the main simulation parameters.

\subsection{Base-Line Resource Allocation Algorithms}

To the best of our knowledge, only one reference paper (Ref. [18]) considers multi-BS coordinated transmission in its resource allocation scheme. However, in Ref. [18], each subchannel of a base station is shared with more than one user. Hence, the scenario considered in our paper that a single user occupies subchannels from multiple BSs cannot be supported. Therefore, it is not appropriate to compare the proposed scheme with Ref. [18] at the moment. In this subsection, two algorithms are used as a base-line for comparison purposes:

Comparison algorithm 1: Proportional Fair algorithm without considering base station coordination (PF-NBSC)

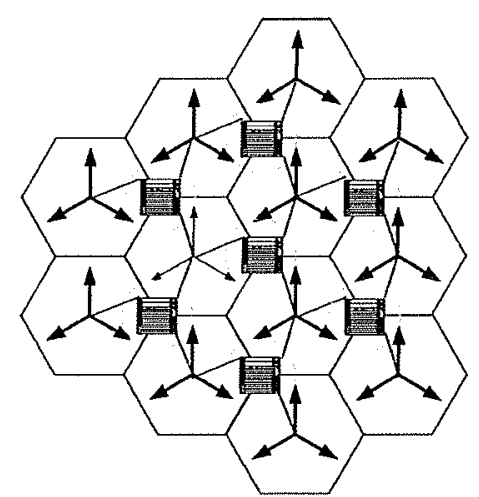

Fig. 2 Structure of the clustered coordinated cellular network. 
Table 1 Simulation parameters.

\begin{tabular}{ll}
\hline Cell Parameters & 12 \\
\hline Number of BSs & $500 \mathrm{~m}$ \\
Cell radius & $43 \mathrm{dBm}$ \\
Maximum power in BS & \\
\hline OFDMA Parameters & $2 \mathrm{GHz}$ \\
\hline Carrier frequency & $10 \mathrm{MHz}$ \\
Bandwidth & $15 \mathrm{KHz}$ \\
Subcarrier spacing & $15.36 \mathrm{MHz}$ \\
Sampling frequency & 1024 \\
FFT size & 50 \\
Number of subchannels & \\
\hline Channel Model & $\mathrm{L}=128.1+37.6 \log _{10} \mathrm{~d}, \mathrm{~d} / \mathrm{km}$ \\
\hline Path loss (dB) & $8 \mathrm{~dB}$ \\
Large-scale fading factor variance & $50 \mathrm{~m}$ \\
Large-scale fading correlation distance & $\mathrm{SCME}[27]$ \\
Small-scale fading &
\end{tabular}

The resources in each BSS are allocated independently without any coordination. The subchannel assignment is done as follows:

- Step 1: For each subchannel, calculate the priority of each MS, which is defined as $\operatorname{Pr}_{i}^{k}(t)=R_{i}^{k}(t) / \vec{R}_{i}(t)$, where $i$ is the MS index, and $k$ is the index of the subchannel.

- Step 2: Subchannel $k$ is allocated to the MS $i^{*}$ with the highest priority.

- Step 3: Go back to step 1 until all subchannels have been allocated.

Comparison algorithm 2: CSA based algorithm without considering base station coordination (CSANBSC)

The subchannel assignment strategy of CSA-NBSC is based on the proposed CSA-BSC algorithm as shown in Eq. (22). Hence, the resources in different BSSs belonging to the same cluster are allocated in a cooperative way. However, in the CSA-NBSC algorithm, the constraint that each MS can only be served by its serving BSS is added, which means multi-BSS coordinated transmission is not allowed.

\subsection{Simulation Results}

In this section, the cell-edge average throughput, the cellaverage throughput and the system faimess metrics are evaluated for the CSA-BSC, CSA-NBSC and PF-NBSC algorithms. The cell-edge performance is evaluated by averaging the performances of the $5 \%$ weakest MSs, while the cellaverage performance is obtained by averaging the throughput of the entire set of MSs within the cell. The fairness index is defined based on [28].

Figure 3 shows the cell-edge average throughput per MS for the three different resource allocation algorithms considered in this paper. It can be seen that the proposed CSA-BSC algorithm achieves the highest cell-edge average

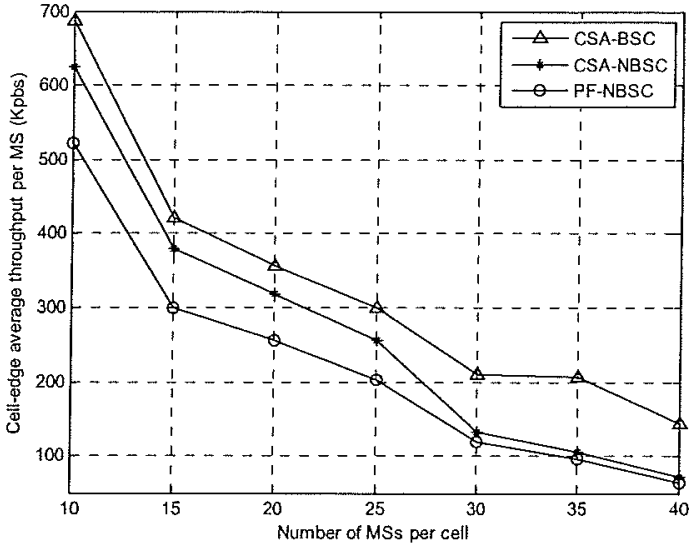

Fig. 3 Cell-edge average throughput vs, number of MSs per cell.

Table 2 Proportion of the MSs served by base station coordinated transmission in CSA-BSC algorithm vs. number of MSs per cell.

\begin{tabular}{llllllll}
\hline Number of MSs & 10 & 15 & 20 & 25 & 30 & 35 & 40 \\
\hline Proportion (\%) & 24 & 18 & 20 & 23 & 18 & 20 & 19
\end{tabular}

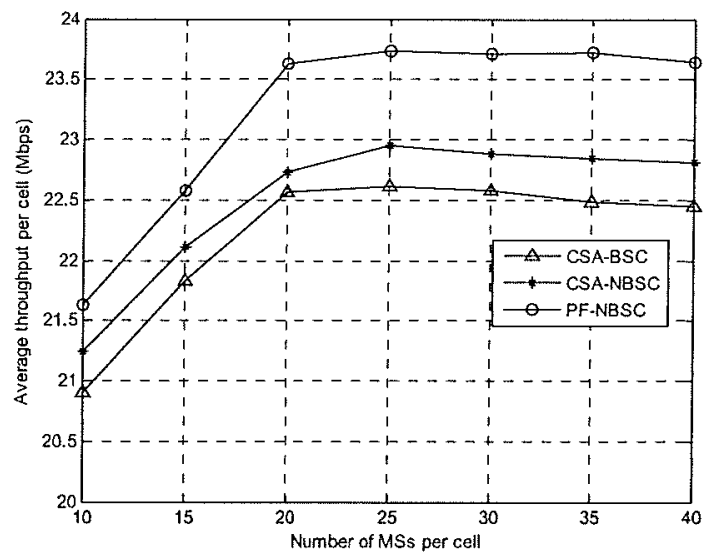

Fig. 4 Average throughput per cell vs. number of MSs per cell.

throughput. When base station coordinated transmission is not supported, each MS can only communicate with its serving BSS, e.g., CSA-NBSC and PF-NBSC. Compared with the PF-NBSC algorithm, the cell-edge average throughput of CSA-NBSC is improved by 9.5 to $26.8 \%$, since the effect of major ICI from neighboring BSS is considered in the channel assignment strategy of the CSA-NBSC algorithm as shown in (20) and (22). When base station coordinated transmission is supported, the cell-edge average throughput raised by the proposed CSA-BSC algorithm is 31.4 to $120.6 \%$ more than the PF-NBSC scheme. As shown in Table 2, the proportion of the MSs served by base station coordinated transmission in the CSA-BSC algorithm is around from $20 \%$, which means about $20 \%$ percent of MSs occupy subchannels from multiple BSs.

Figure 4 shows the cell-average throughput of the three resource allocation algorithms considered in this paper as a 


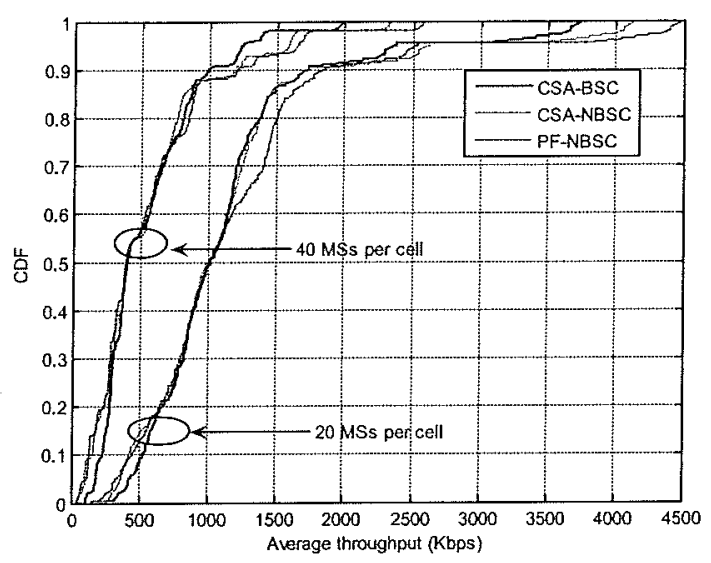

Fig. 5 The CDF of MS throughput vs. number of MSs per cell.

function of the number of MSs per cell. We can see that the cell-average throughput of the PF-NBSC algorithm outperforms that of the CSA-NBSC and the CSA-BSC algorithms. Compared with the PF-NBSC algorithm, the cell-average throughput of the CSA-NBSC and the CSA-BSC algorithms is reduced by $1.8 \%$ and $3.3 \%$, respectively, when the number of MSs per cell is small, i.e. less than 15. When the number of MSs is large, the average throughput of the PF-NBSC algorithm is 3.0 to $3.8 \%$ more than the CSA-NBSC algorithm and 4.4 to $5.3 \%$ more than the CSA-BSC algorithm. However, the payoff for this higher cell-average throughput of the PF-NBSC algorithm is a significant decrease in the celledge average throughput, which can be seen from Fig. 3 .

Figure 5 shows the cumulative density function (CDF) of the average throughput of all the users in the system for the CSA-BSC, CSA-NBSC and PF-NBSC algorithms vs. number of MSs per cell. It can be observed that the mean throughput of PF-NBSC is greater than that of CSA-NBSC and CSA-BSC. However, the highest cumulative probability of low throughput is obtained in PF-NBSC. It means that PF-NBSC leads to higher cell throughput but worse performance for the cell-edge users. In addition, CSA-BSC has the best cell-edge performance, since base station coordinated transmission is supported. The trend of the results is similar to that of Fig. 3 and Fig. 4.

Finally, Jain's Fairness Index (JFI) is investigated as a fairness measure of the algorithm for resource allocation [28]. The fairness index (FI) is calculated based on MSs average throughput. In this paper the FI is defined as

$$
\mathrm{FI}=\left(\sum_{m=1}^{M} \vec{R}_{m}\right)^{2} /\left(M \sum_{m=1}^{M} \bar{R}_{m}^{2}\right) .
$$

Figure 6 shows that CSA-BSC achieves the best fairness performance among the three resource allocation algorithms considered in the paper. The fairness gain increases as the number of MSs per cell increases. That is because the subchannel assignment strategy in the CSA$\mathrm{BSC}$ algorithm coordinates the transmission of neighboring BSSs, and such coordinated transmission can guarantee

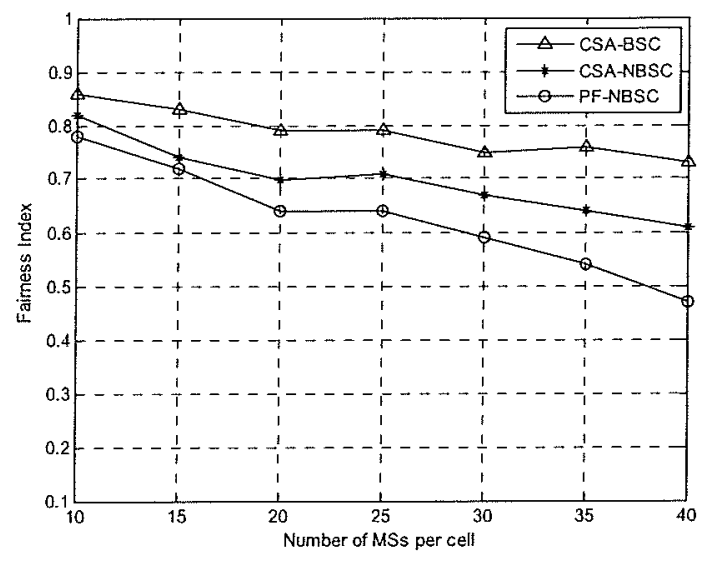

Fig. 6 Fairness index vs. number of MSs per cell.

higher cell-edge throughput as shown in Fig. 3.

\section{Conclusion}

In this paper, we consider the downlink of a coordinated cellular OFDMA networks. A utility-based radio resource allocation algorithm is proposed to support base station coordinated transmission and further improve the cell-edge performance. Firstly, with the assumption of global coordination of all the base station sectors in the network, a coordinated subchannel assignment algorithm is proposed, which maximizes the users' sum utility function with respect to the user average throughput. Then, by dividing the available base station sectors into a number of disjoint coordinated clusters, a reduced-feedback algorithm for subchannel assignment is proposed for practical use. Simulation results demonstrate that the reduced-feedback coordinated subchannel assignment offers substantial cell-edge performance improvement and system fairness improvement in aggressive frequency reuse cellular networks, with acceptable degradation of cell-average performance.

The results in this paper assume perfect channel state information. In future work, the impact of imperfect channel state information on the performance of the proposed algorithms will be addressed. In addition, the joint power control and subchannel allocation problem with advanced multi-antenna transmission methods will be considered.

\section{Acknowledgments}

This work is supported by the Project 6100116 of National Natural Science Foundation of China, National 863 Project 2009AA011506, State Emphasis Special Project 2009ZX03003-011-02, the Project 60872048 of National Natural Science Foundation of China, the Sino-Sweden Project (No. 2008DFA12110), The Swedish Agency for Innovation Systems (VINNOVA), No. P33514-1.

\section{References}

[1] 3GPP TR 25.814 ×7.1.0, "Physical layer aspects for evolved UTRA 
(Release 7)," Sept. 2006.

[2] 3GPP TR 36.814 v1.0.1, "Further advancements for E-UTRA physical layer aspects (Release 9)," March 2009.

[3] G. Boudreau, J. Panicker, N. Guo, R. Chang, N. Wang, and S. Vrzic, "Interference coordination and cancellation for $4 \mathrm{G}$ networks," IEEE Commun. Mag., vol.47, no.4, pp.74-81, April 2009.

[4] R.G. Vaughan, "On optimum combining at the mobile," IEEE Trans. Veh. Technol., vol.37, no.4, pp.181-188, Nov. 1988.

[5] J.G. Andrews, "Interference cancellation for cellular systems: A contemporary overview," IEEE Wireless Commun. Mag., vol.12, no.2, pp.19-29, April 2005.

[6] M.K. Karakayali, G.J. Foschini, and R.A. Valenzuela, "Network coordination for spectrally efficient communications in cellular systems," IEEE Wireless Commun. Mag., vol.13, no.4, pp.56-61, Aug. 2006.

[7] W. Choi and J.G. Andrews, "Downlink performance and capacity of distributed antenna systems in a multicell environment," IEEE Trans. Wirel. Commun., vol.6, no.1, pp.69-73, Jan. 2007.

[8] H. Zhang and H. Dai, "Cochannel interference mitigation and cooperative processing in downlink multicell multiuser MIMO networks," EURASIP J. Wireless Commun. and Networking, vol.2004, no.2, pp.222-235, Dec. 2004.

[9] J. Zhang, R. Chen, J. Andrews, A. Ghosh, and R. Heath, "Networked MIMO with clustered linear precoding," IEEE Trans. Wireless Commun., vol.8, no.4, pp.1910-1921, April 2009.

[10] C.Y. Wong, R.S. Cheng, K.B. Letaief, and R.D. Murch, "Multiuser OFDM with adaptive subcarrier, bit, and power allocation," IEEE $\mathbf{J}$ Sel. Areas Commun., vol.17, no.10, pp.1747-1758, Oct. 1999.

[11] K. Seong, M. Mohseni, and J.M. Cioffi, "Optimal resource allocation for OFDMA downlink systems," Proc. IEEE ISIT’06, pp.13941398, July 2006.

[12] J. Huang, V.G. Subramanian, R. Agrawal, and R.A. Berry, "Downlink scheduling and resource allocation for OFDM systems," IEEE Trans. Wirel. Commun., vol.8, no.1, pp.288-296, Jan. 2009.

[13] G. Li and H. Liu, "Downlink radio resource allocation for multicell OFDMA system," IEEE Trans. Wirel. Commun., vol.5, no.12, pp.3451-3459, Dec. 2006.

[14] M.C. Necker, "A graph-based scheme for distributed interference coordination in cellular OFDMA networks," Proc. IEEE VTC'08, pp.713-718, May 2008.

[15] S. Pietrzyk and G.J. Janssen, "Radio resource allocation for cellular networks based on OFDMA with QoS guarantees," Proc. IEEE GLOBECOM'04, pp.2694-2699, Nov. 2004.

[16] L. Venturino, N. Prasad, and X. Wang, "Coordinated scheduling and power allocation in downlink multicell OFDMA networks," IEEE Trans. Veh. Technol., vol.58, no.6, pp.2835-2848, July 2009.

[17] L. Chen, W. Chen, X. Zhang, Y. Zhang, C. Xiong, and D. Yang "Inter-cell coordinated resource allocation for mobile WiMAX system," Proc. IEEE WCNC’09, pp.1-6, April 2009.

[18] Ronald Y. Chang, Zhifeng Tao, Jinyun Zhang, and C.-C. Jay Kuo, "Multicell OFDMA downlink resource allocation using a graphic framework," IEEE Trans. Veh. Technol., vol.58, no.7, pp.34943507, Sept. 2009

[19] A. Papadogiannis, E. Hardouin, and D. Gesbert, "Decentralising multicell cooperative processing: A novel robust framework," EURASIP J. Wireless Commun. and Networking, vol.2009, pp.110, 2009.

[20] A. Papadogiannis, D. Gesbert, and E. Hardouin, "A dynamic clustering approach in wireless networks with multi-cell cooperative processing," Proc. IEEE ICC'08, pp.4033 4037, May 2008

[21] F. Boccardi and H. Huang, "Limited downlink network coordination in cellular networks," Proc. IEEE PIMRC'07, pp. 1-5, Sept. 2007.

[22] M. Kamoun and L. Mazet, "Base-station selection in cooperative single frequency cellular network," Proc. IEEE SPAWC"07, pp.1-5, June 2007.

[23] P. Marsch and G. Fettweis, "A framework for optimizing the uplink performance of distributed antenna systems under a constrained backhaul," Proc. IEEE ICC'07, pp.975-979, June 2007.

[24] A.J. Goldsmith and S.-G. Chua, "Variable-rate variable-power MQAM for fading channels," IEEE Trans. Commun., vol.45, no. 10, pp.1218-1230, Oct. 1997.

[25] G. Song and Y. Li, "Cross-layer optimization for OFDM wireless networks-part II: Algorithm development," IEEE Trans. Wirel. Commun., vol.4, no.2, pp.625-634, March 2005.

[26] C.Y. Wong, C.Y. Tsui, R.S. Cheng, and K.B. Letaief, "A real-time subcarrier allocation scheme for multiple access downlink OFDM transmission," Proc. IEEE VTC'99, vol.2, pp.1124-1128, Sept. 1999.

[27] D.S. Baum, J. Salo, G. Del Galdo, M. Milojevic, P. Kyösti, and J. Hansen, "An interim channel model for beyond-3G systerns," Proc. IEEE VTC'05, pp.3132-3136, May 2005.

[28] A. Sang, X. Wang, M. Madihian, and R.D. Gitlin, "A flexible downlink scheduling scheme in cellular packet data systems," IEEE Trans. Wirel. Commun., vol.5, no.3, pp.568-577, March 2006.

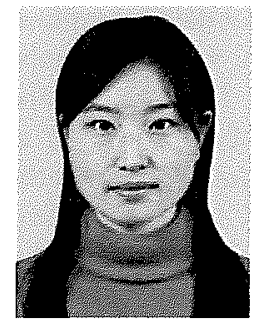

Jingya $\mathrm{Li}$ received her B.S. degree in Telecommunication Engineering from Beijing University of Posts and Telecommunications (BUPT) in 2008. She was a master student major in communication and information system in BUPT during Sept. 2008 to Jun. 2010. Now she is a Ph.D. student at Chalmers University of Technology since Oct. 2010. Her research interests cover radio resource management in future mobile communication network.

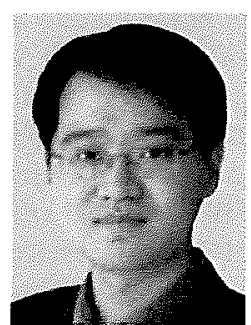

Xiaodong $\mathrm{Xu}$ received his B.S. degree in information and communication engineering 2001 and M.S. degreein Communication and Information System 2004 both from Shandong University. He received his Ph.D. degrees of Circuit and System in Beijing University of Posts and Telecommunications (BUPT). He is currently Associate Professor in BUPT. His research interests cover advanced mobile communication systems and the key technologies, including radio resource management, coordinated multipoint for next generation mobile system and standardization of Enhanced $3 \mathrm{G}$ systems.

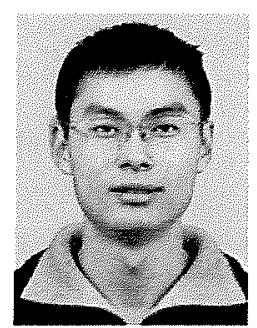

Xin Chen

received his B.S, degree in Telecommunication engineering from Huazhong University of Science and Technology in 2008. Now he is a Ph.D. Candidate in BUPT. His research interests cover radio resource management in future mobile communication network. 


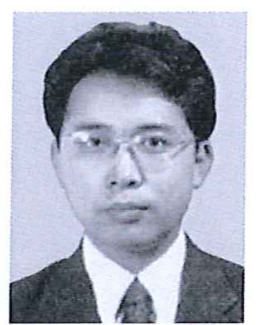

Xiaofeng Tao received the B.S. degree in electrical engineering from Xi' an Jiaotong University, China, in 1993, and M.S.E.E. and Ph.D. degrees in telecommunication engineering from Beijing University of Posts and Telecommunications in 1999 and 2002, respectively. $\mathrm{He}$ was a research engineer working in the Posts and Telecommunications Industry Company of China (PTIC) from 1993 to 1996. He is currently a professor in BUPT and a group leader of the TDD Special Working Group of China 863 FuTURE Program. His research interests cover techniques for B3G, such as space-time coding, MIMO, novel cell structures, and intelligent group handover mode.

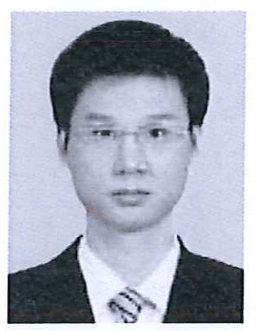

Hui Zhang received his B.S. degree in Applied Mathematics in 2005, and M.S. degree in Telecommunication Engineering in 2007, both from Beijing University of Posts and Telecommunications (BUPT). Now he is a Ph.D. Candidate in BUPT. His research interests cover the key techniques of the $\mathrm{B} 3 \mathrm{G} / 4 \mathrm{G}$ systems.

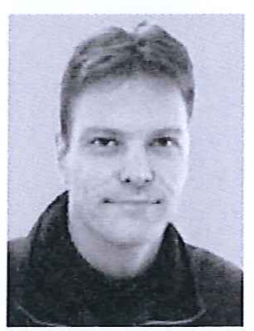

Tommy Svensson received the M.S. degree in Engineering Physics 1994 and Ph.D. degree in Information Theory 2003, both from Chalmers University of Technology, Sweden. He has contributed to the development of the 3GPP LTE standard through the European collaboration projects EU FP6 WINNER, WINNER II, and CELTIC WINNER+. He is currently Associate Professor in Communication Systems at Chalmers, where he is leading the research on air interface and microwave backhauling technologies for IMT Advanced and beyond. He has also been working at Ericsson $\mathrm{AB}$ with core networks, radio access networks and microwave transmission products. His main expertise is in design and analysis of physical layer algorithms and multiple access schemes for multi-cellular wireless communication systems, but he is also experienced in higher layer design of wireless communication systems. He is a senior member of IEEE.

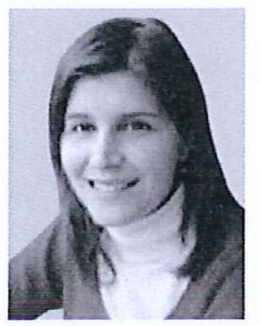

Carmen Botella received the Ingeniero de Telecomunicación degree from the Universidad Politécnica de Valencia, Spain, in 2003, and the Ph.D. degree from the Universidad Politécnica de Valencia, Spain, in 2008. During her Ph.D., she worked as a research assistant within the Institute of Telecommunications and Multimedia Applications (iTEAM) of the Universidad Politécnica de Valencia, Spain. In 2006, she was a visiting researcher in the Eurecom Institute (Sophia-Antipolis, France) under the supervision of Professor David Gesbert. In 2009, she joins the Communications Systems and Information Theory group of Chalmers University of Technology as a Postdoc. 